\title{
Using Wind Shock-Waves and Turbulence as a Soft Attrition Capability against a Smart Adversary Team of UAVs
}

\author{
$\underline{\text { V. Ivancevic }}^{\text {a }}$, S. Boswell, D. Gossink \\ ${ }^{a}$ Decision Sciences, JOAD, DST-Group \\ Email: Vladimir.Ivancevic@dst.defence.gov.au
}

\begin{abstract}
In this paper we propose a new soft-attrition capability: using wind shock-waves and turbulence against a smart adversary team of unmanned aerial vehicles (UAVs) within a confined space in an urban environment. A 3D UAV Simulator has been developed in Decision Sciences, JOAD, DST-Group, based on the theoretical foundation for controlling swarms of UAVs. A particular version of the simulator (used in this paper) was designed for indoor navigation of a team of UAVs with the objective to sustain their stable flight under wind turbulence conditions. The core of this indoor UAV simulator is a nonlinear, highly-robust, fuzzy-logic control based, smart individual collision-avoidance system for each UAV, which results in the flocking behavior of a whole team under threat. The simulator uses two types of sensors: radars-on-a-chip and sonars, 6 radars and 6 sonars per UAV, one radar and one sonar per side of the UAV's bounding box. The collision avoidance system was designed by combining (via logical And) the speed and distance fuzzy-logic If-Then rules of the form:
\end{abstract}

If an object is approaching from Front with high speed Then move Backward with high speed, And If an external object is very close in Front, Then move Backward with high speed, etc.

To counteract this smart team of adversary UAVs, a new soft-attrition capability has been developed in the form of a wind shock-wave field (which develops into a tornado-type wind turbulence inside a confined space), defined by the Tanh-solution of the nonlinear Schrödinger equation. The wind shock-wave field represents a fast-traveling train of non-dissipating solitons (like a 1-dimensional train of neural impulses running down the neural fibre, or a 2-dimensional series of tsunamis caused by an underwater earthquake and fast-flowing along the ocean from one shore to the other). This non-dissipating wind wave depends on two parameters, amplitude and frequency, which can be changed at will. The qualitative analysis presented in this paper shows that even the smart team of bird-like UAVs with superb collision-avoidance system can be blown away by the wind shock-waves (with the amplitude that is many times higher than the thrust capabilities of the individual UAVs) and the tornado-like turbulence caused in the confined environment.

Keywords: Smart UAVs, collision-avoidance system, wind shock-waves and turbulence, soft attrition 


\section{INTRODUCTION}

The current revolution in the availability of technology is delivering drones into the hands of the general public via the internet and local retailers. Small airborne drones can be controlled by almost any person, and can be directed into many locations, possibly without permission and potentially for nefarious purposes (see Custers (2016) and the references therein). There will be many locations for which it is desirable to prevent drone access, and the suitability of prevention methods will need to be explored. This paper explores, via a simulation, the potential of a simple method of controlling access by adversary drones in the form of wind shock-waves. Wind control is explored as electronic methods may not be suitable for applications in areas where electronics are in use.

A 3D UAV Simulator (see Figures 1-4) has been developed in Decision Sciences, Joint and Operations Analysis Division (JOAD), Defence Science and Technology-Group (Australia), based on the theoretical foundation for controlling swarms of unmanned ground and aerial vehicles (UGVs and UAVs, respectively) presented in Ivancevic and Yue (2016). Two versions of the visually-realistic simulator of moving a swarm of UAVs within a certain urban environment, both outdoors and indoors, have been developed in the dot.Net 4.6 framework, combining the $\mathrm{C \#}$ and $\mathrm{C}++$ languages as a single project/solution in MS Visual Studio 2015, using the Irrlicht $3 \mathrm{D}$ graphics engine for various urban environmental maps and the Bullet 3D physics engine for gravity, inertia and collision detection.

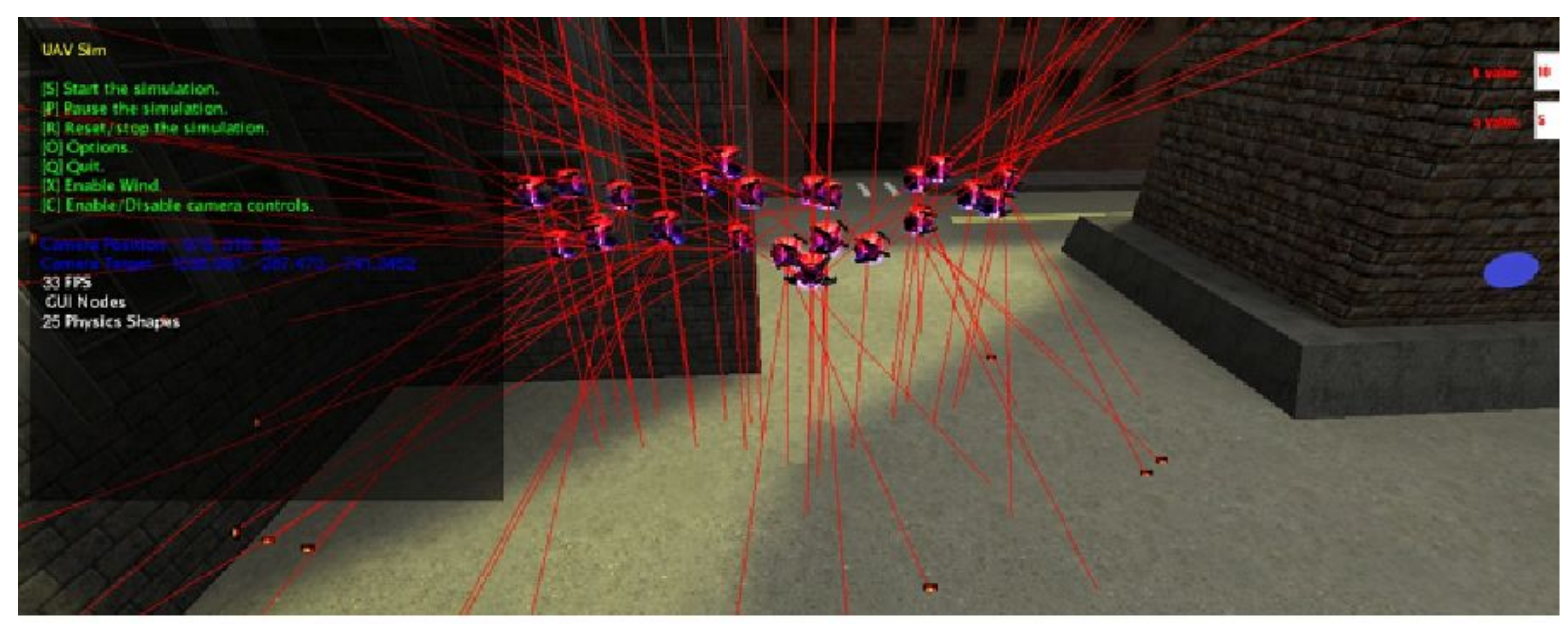

Figure 1. A snapshot from DST-Group's 3D UAV Simulator, depicting a small swarm of adversary UAVs (compare with Schoellig et al. (2012)) entering a city block. Each UAV has its own collision avoidance system based on fuzzy-logic controlled sonar sensors (red lines; see Ivancevic et al. (2017)). A blue ellipsoid on the right represents the "wind canon" that triggers the shock-wave type wind turbulence (defined in Ivancevic et al. (2017)), which is controlled by the user (as a "human-in-the-loop"). This initial figure shows the start of the animation: a free flight of the Red UAVs within Blue's confined urban space. Upon triggering the wind turbulence, UAVs immediately respond by grouping and landing (we refer to this behavior as fuzzy entanglement).

The simulator was designed for indoor navigation of a team of UAVs with the objective to sustain their stable flight under wind turbulence conditions. So, the core of the simulator is a nonlinear, highly-robust, fuzzy-logic control based, smart individual collision-avoidance system for each UAV (as described in Ivancevic et al. (2017)), which results in flocking behavior by a whole team under threat. The simulator uses two types of sensors: radars-on-a-chip and sonars, 6 radars and 6 sonars per UAV, one radar and one sonar per side of the UAV's bounding box. The collision avoidance system was designed by combining the speed and distance fuzzy-logic If-Then rules, as:

If an object is approaching from Front with high speed Then move Backward with high speed, And If an external object is very close in Front, Then move Backward with high speed, etc. 
In this way defined, smart collision-avoidance system on each UAV, generates a group behavior called fuzzy entanglement (see Ivancevic et al. (2017) for additional technical details).

\section{WIND SHOCK-WAVES AND TURBULENCE MODEL}

A $(1+1)$ complex-valued wind shock-wave function which has been defined as the following Tanh-solution of the nonlinear Schrödinger equation (see Ivancevic (2010), Ivancevic (2011), Ivancevic et al. (2017)):

$$
\psi(x, t)=a \sqrt{\frac{1}{V(x)}} \operatorname{Tanh}(x-k t) \operatorname{Exp}\left[\mathrm{i}\left(k x-\frac{t}{2}\left(k^{2}-a^{2}\right)\right)\right],
$$

where $\mathrm{i}=\sqrt{-1}$ is the imaginary unit, $V(x)=\operatorname{Sech}(x)$ is the bell-shaped potential field, $t$ is time, $x$ is the spatial coordinate, and $a$ and $k$ are parameters (discussed below). This is also Eq. (5) from Ivancevic et al. (2017) as a part of the general wind turbulence model, which includes 9 more equations similar to (1), mostly based on Jacobi sine and cosine functions, all equations having the same exponential part and all representing quantum turbulence, or controlled turbulence (as opposed to uncontrollable Navier-Stokes-based turbulence; for technical details see Ivancevic and Reid (2012) and the references therein).

This is our theoretical model for the $2 \mathrm{D}$ wind shock-wave in the complex plane. For the purpose of a 3D simulation in an urban environment (as a part of the 3D UAV simulator), the Tanh-soliton Eq. (1) has been simplified into the following real form (in the constant potential field and generalized to 3D with $(x, y, z)$ coordinates), defining the following three Cartesian wind forces: ${ }^{1}$

$$
\begin{aligned}
& F_{x}=30 a \sqrt{\operatorname{Cosh}(x)} \operatorname{Tanh}(x-k t) \operatorname{Sin}\left(k x-0.5 t\left(k^{2}-a^{2}\right)\right), \\
& F_{y}=5 a \sqrt{\operatorname{Cosh}(y)} \operatorname{Tanh}(y-k t) \operatorname{Sin}\left(k y-0.5 t\left(k^{2}-a^{2}\right)\right), \\
& F_{z}=30 a \sqrt{\operatorname{Cosh}(z)} \operatorname{Tanh}(z-k t) \operatorname{Sin}\left(k z-0.5 t\left(k^{2}-a^{2}\right)\right),
\end{aligned}
$$

with the following default values of the wind shock-wave parameters $a$ (amplitude) and $k$ (frequency/wave number): $a=5 m, \quad k=10 \mathrm{~Hz}$, which are built in the wind simulator. The amplitude for the $F_{y}$ value is smaller since we are actually dealing with the 2D shock-wave. We remark that, as usual in 3D graphics, the vertical axis is $Y$, rather than $Z$ (as usual in geometry).

In the present study, a device called the "wind canon" has been included to trigger wind shock waves as a soft attrition capability against adversary UAVs. A displacement of a UAV in any of the three Cartesian directions is a result of two main factors:

1. Its own collision-avoidance based motion (as a member of a swarm), and

2. Blowing (a sudden push/pull) by the super-fast train of wind shock-waves, which produces a turbulence field inside confined urban environments.

Eqs. (1)-(2), at every time instant, produce a 3D shock-wave spreading in $(x, y, z)$-directions. In each Cartesian direction, its profile is given by Figure 2, which shows the dependence of the wave profile on the variation of the amplitude parameter $a$.

\section{NUMERICAL DYNAMICS AND QUALITATIVE ANALYSIS}

In this section we provide a supplementary, model-supportive analysis of the position data of the UAVs extracted from the 3D simulator. Since it necessarily neglects all "hidden variables" related to collision-avoidance and Bullet physics, this analysis of the synthetic data is not meant to be conclusive but rather indicative of the correctness of the competitive model of the wind turbulence against smart collision-avoidance, within the simulated urban environment.

The preliminary data analysis (performed in Wolfram Mathematica) suggests that numerical inverse dynamics (given the positional data, calculate the forces) - cannot be successfully performed by double timedifferentiation of the empirical UAV-position data, for two reasons: (i) the raw position data is too noisy

\footnotetext{
${ }^{1}$ These forces represent a simplified version of Eq. (1): using the bell-shaped sech potential $(V(x)=\operatorname{Sech}(x))$, simplifying the complex exponent with a real sinus, and adding the third, vertical dimension to the original $2 \mathrm{D}$ shock-wave.
} 

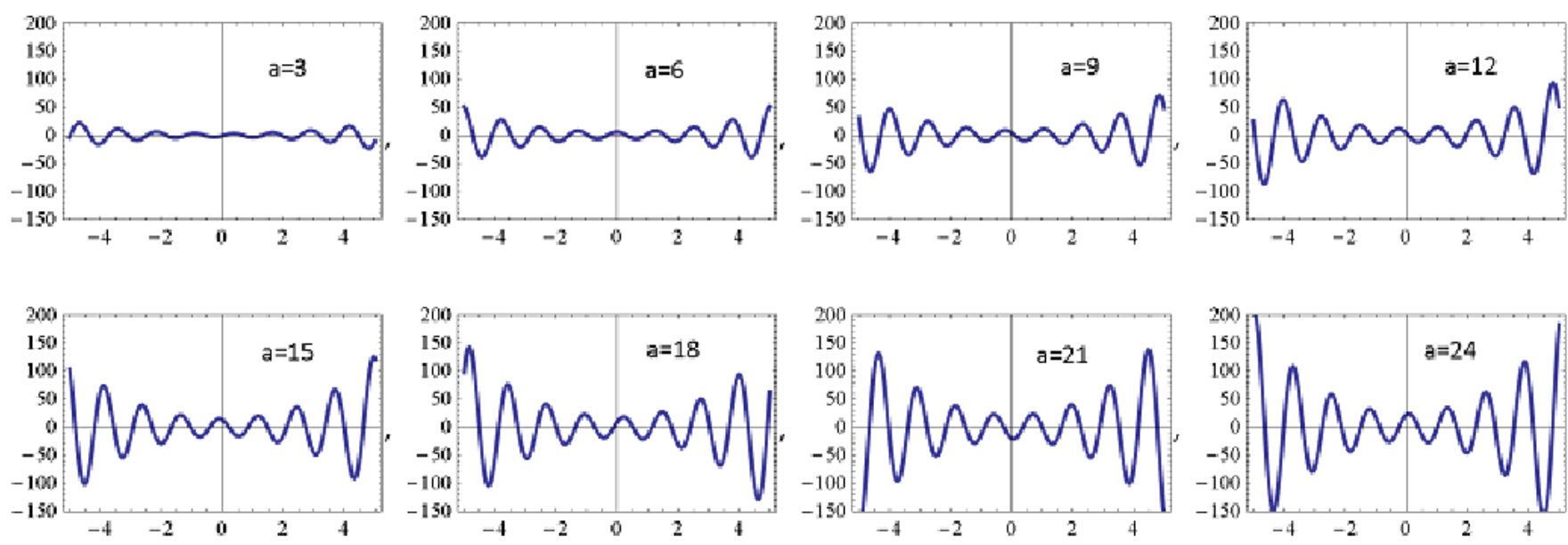

Figure 2. Instant profile of the wind shock-wave with different values of the amplitude $a$. All snapshots are taken with the fixed frequency $k=5 \mathrm{~Hz}$ at the time $\mathrm{t}=2 \mathrm{sec}$, within the $10 \mathrm{~m}(-5,+5)$ range.

to start with (see Figure 3 - left), and (ii) numerical differentiation is a noise-amplification procedure itself. Therefore, the initial noise would amplify to the point that it is meaningless. Only the semi-analytical double differentiation might work well on a sufficiently smooth $\left(C^{2}\right.$, i.e., at least twice-differentiable) data, which is not our case.

On the other hand, numerical forward dynamics (given the forces, calculate the positions) - can be successfully performed by double time-integration of the empirical wind forces data extracted from the 3D Simulator, since numerical integration is a noise-reduction procedure (emulating a low-pass filter), so even from noisy raw forces we can calculate quite smooth velocities (times mass) and subsequently positions (times mass). In such a way calculated dimensionless UAV positions, mostly agree as a general trend with the extracted positions (see Figure 3). Such trend agreements between predicted and actual positions of UAVs we call "visual crosscorrelations". Predicted UAV positions basically show what would be the actual positions if there were not collision avoidance systems on the UAVs.
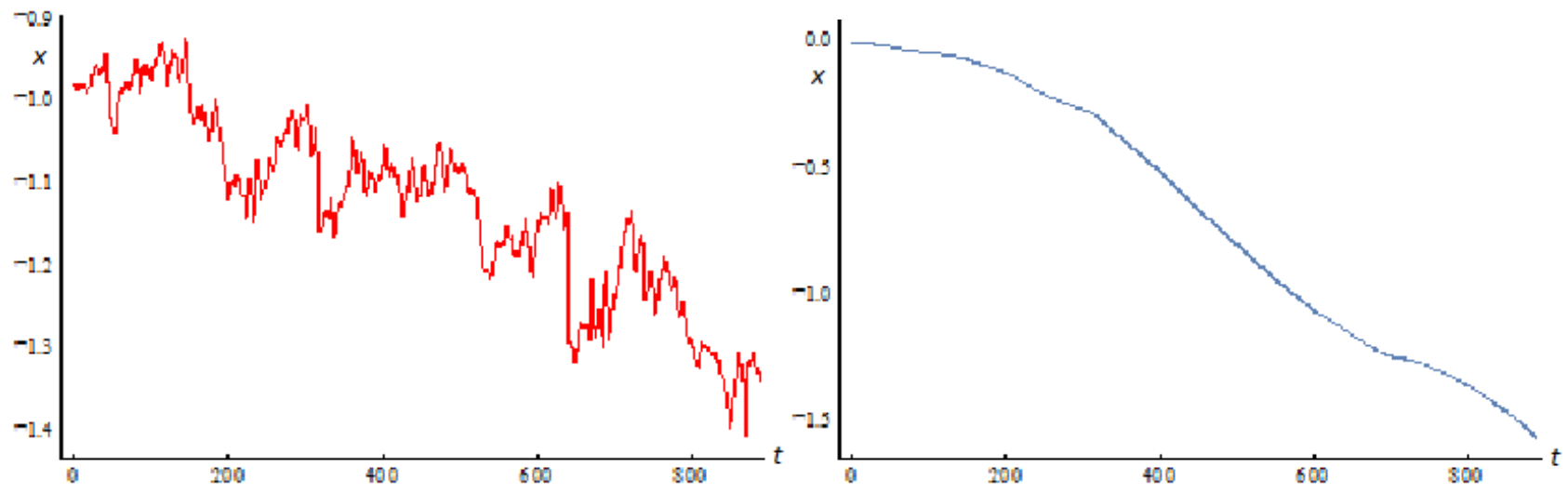

Figure 3. Raw UAV position data in 890 discrete time steps (left) and double-integrated force data (right). The required scaling can be interpreted as an unknown radius-dependent mass (which is a parameter in the Bullet physics engine), while the required vertical shift can be interpreted as two integration constants: initial position and velocity. Finally, the double-integrator provides a smooth output (it naturally behaves like a lowpass filter), while the UAV dynamics is highly-fluctuated, as a response of its collision-avoidance system to the wind shock-wave within the confined urban environment.

Encouraged by the preliminary analysis, we have performed numerical Forward Dynamics: (Forces $\left\{f_{n}\right\}$ ) $\Longrightarrow$ 
(Motions $\left\{x_{n}\right\}$ ), starting with the wind force data $f_{n}$ from the input CSV-file, and calculating the predicted UAV-positions $x_{n}$, using the double Euler integration, as (see Chapra and Canale (2009)):

$$
v_{n+1}=v_{n}+f_{n} d t, \quad x_{n+1}=x_{n}+v_{n} d t
$$

where $v_{n}$ are the calculated velocities (times mass) and $d t$ is the time-step averaged from the input CSV-file. The Fortran 90 code (available on request) was developed to read the force data $f_{n}$ from the input CSV-file, perform the Euler integration given by Eq. (3) along the three Cartesian axes $(x, y, z)$ and output both input and output data (all scaled-down by 1000 for easier interpretability) into the output CSV-file for the easy plotting in Excel.

Firstly, we compare predicted and simulated actual positions for a single UAV. We define the "visual crosscorrelations" as "mostly agree as a visual trend." We can see on the graphs that the same behavior often appears, with corresponding regions that are flat or have the same slope, indicating the same visual trend. These regions are circled."

This qualitative analysis of synthetic data has only an indicative value and is not conclusive. The simulated environment is too complex to be conclusively represented by any qualitative relation.

A sample chart with default wind amplitude and frequency is shown in Figure 4. Similar charts are obtained with doubling the amplitude and with doubling the frequency.
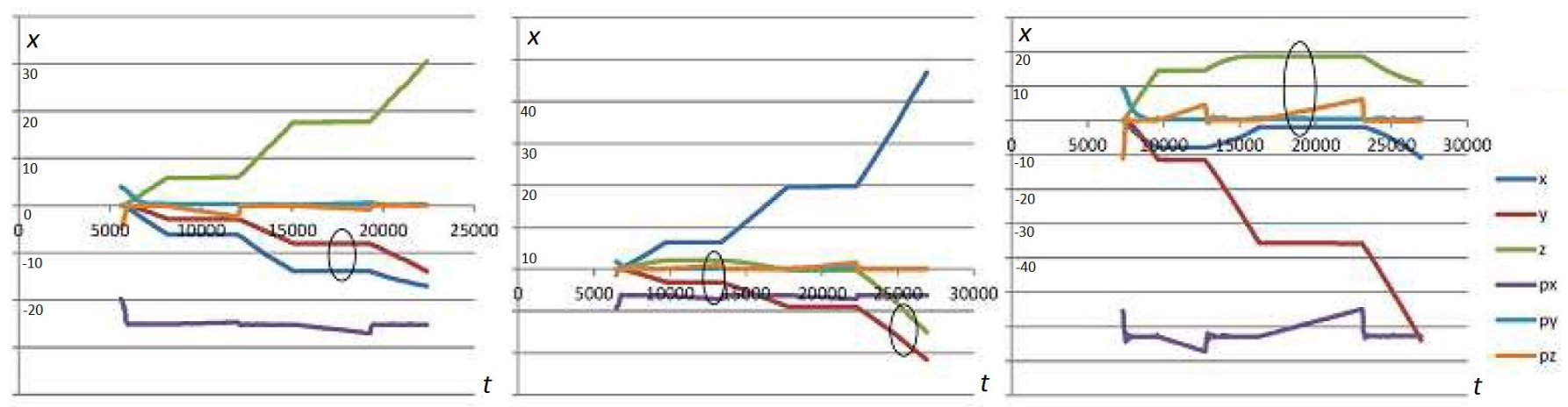

Figure 4. Single UAV: qualitative comparison of the actual UAV-position vectors $\left(p_{x}, p_{y}, p_{z}\right)$ and predicted positions $(x, y, z)$ calculated by double-integrating the force data in three sample simulations with default values of both amplitude and frequency of the wind shock-wave field. The labels on the vertical axes denote displacement in meters.

Secondly, we compare predicted and actual positions for three UAVs. All "visual cross-correlations," showing that signals mostly agree as visual trends, are circled as in the previous subsection.

A sample chart with default wind amplitude and frequency is shown in Figure 5. Similar charts are obtained with doubling the amplitude and with doubling the frequency. We remark that doubling the wind amplitude has more effect than doubling the wind frequency (provided in the longer paper version).

From these charts, we can see that a number of "visual cross-correlations" have been identified, more so in the case of three UAVs. The simulated behavior shows some features of the analytically predicted behavior, but not all. Analytically predicted behavior does not take into account the intelligence of the adversary UAVs: they group together and land down as soon as the wind is triggered on (this is their fuzzy entanglement, a consequence of the fuzzy-logic based collision-avoidance system). So, all qualitative analysis can be used only for illustrative purposes, and for these purposes this is enough. The proposed conclusion is the following: synthetic model-extracted data analysis shows the capability of the wind turbulence model as a soft-attrition weapon against the team of smart UAVs, each enabled with a sophisticated collision-avoidance system that is far superior than any existing UAV collision-avoidance system (each UAV has 6 radars and 6 sonars combined in a smart nonlinear controller). This smart collision-avoidance system effectively counteracts the wind field. Recall that, in the first place, the 3D UAV simulator has been built to show superior UAV control under turbulent wind conditions. Now, even in these competitive conditions against the smart adversary, the wind 

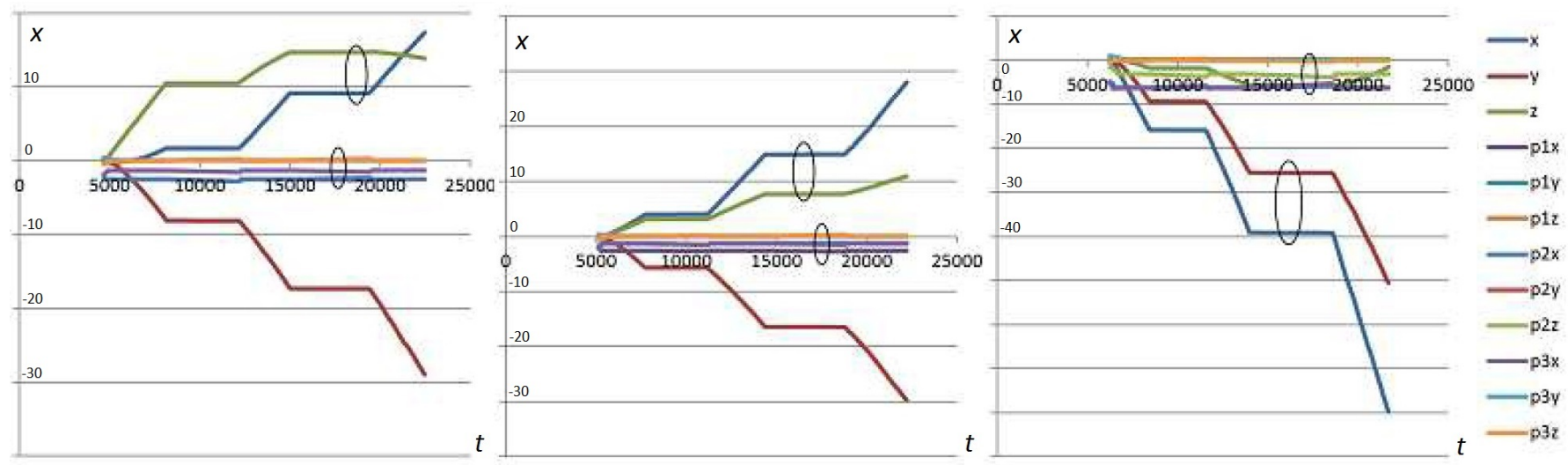

Figure 5. Group of 3 UAVs: Qualitative comparison of the actual UAV-position vectors $\left(p_{x}^{i}, p_{y}^{i}, p_{z}^{i}\right.$; for $i=1,2,3)$ and predicted positions $(x, y, z)$ calculated by double-integrating the force data in three sample simulations with default values of both amplitude and frequency of the wind shock-wave field. The labels on the vertical axes denote displacement in meters.

shock-wave field performs well. A less sophisticated adversary would be simply blown away in the open environment or into the walls in the confined environment.

\section{DISCUSSION}

Nine simulations have been performed with a single UAV and nine with a team of three UAVs (raw data are available on request). These raw data, including wind forces and UAV positions, suggest that the stronger the wind amplitude and/or the frequency the stronger the effect.

There may be a frequency of wind that causes maximal interference with a copter based UAV's flight and control system (if it has no compensator), that is the UAV is blown further away, both in case of a single UAV and a team of UAVs, although in the case of teams the wind-motion relation is more complex due to collision-avoidance interactions. Also, increasing the wind amplitude has a stronger effect than increasing the wind frequency.

The simulated values for wind strength/frequency are only "visually realistic" (i.e., the "visual crosscorrelations" behave as expected), because non-dimensional physical units are used, defined by the Bullet physics engine. ${ }^{2}$

It should be possible to find a resonance frequency, specific to a certain kind of quadcopter, which would interfere with the UAV's navigation system as well, In this way, a UAV would be both control-disabled and blown away. However, this frequency parameter is actually a wave number, that is, frequency both in time $t$ and in space $(x, y, z)$, so the situation is a bit more complex.

In any case, predicted UAV positions (calculated as double time-integrated forces) show what would be the actual positions if there were not collision avoidance systems on the UAVs.

In an open space, the model produces a fast train of blasts, or shock waves, which inside a confined environment produces a field of turbulence which blows away all small objects.

Therefore, the proposed wind turbulence model shows a new capability, an effective soft-attrition weapon against a smart adversary team of UAVs, significantly more intelligent than any low-price commercial UAVs. Performance of the wind turbulence model would be much higher against a less intelligent adversary UAVs.

A very strong version of the standard leaf-blower can be used as a wind cannon against small UAVs. For big UAVs the same design with a stronger turbine could be used. However, this simplistic kind of a wind-blower, no matter how strong, will be damped by the inverse square law: $\left(1 / d^{2}\right)$, where $d$ is the Euclidean standoff distance between the UAV and the wind source. On the other hand, a small and portable jet turbine, which we call a "wind canon/machine gun", would produce a non-dissipative (zero damping) solitons, i.e., traveling

${ }^{2}$ From the visual impression, assuming a UAV mass of the order of magnitude of $1 \mathrm{~kg}$, a human with e.g. $80 \mathrm{~kg}$ should be able to maintain a stable walk through the wind shock-waves even with a double amplitude. This is dependent on the amplitude of the wave generated by the jet turbine (see Figure 2). 
waves with high speed and constant kinetic energy. In other words, the force-field of the wind shock-wave does not depend on the distance between the UAV and the wind source - the wind wave travels through the confined (indoor-like) environment and hits a UAV with the same amplitude and frequency wherever it is currently located. However, at this stage, this is only a hypothetical "wind canon" model, defined by the Tanh-soliton Eqs. (1)-(2).

Therefore, the theoretical wind-force model defined by Eqs. (1)-(2) is showing that in a confined space (within short distances) we have a non-dissipating force field with the full conservation of energy. However, when we go to the physical application, if we use a leaf-blower type of a wind source, then we have to deal with the inverse square type of dissipation of energy.

Inside a confined space (like an aircraft hanger) a wind turbine, with the action defined by the model Eqs. (1)(2) can generate a tornado-type wind turbulence that would smash all UAVs into the walls, regardless of their weights. In an open space (outside of the hanger) the model produces a fast train of shock-waves (wind blasts), depending on the frequency parameter $k$, which will repel the UAVs like a fast series of punches $(\mathrm{k}=10 \mathrm{~Hz})$.

\section{CONCLUSION}

The 3D UAV simulator shows that even a smart team of UAVs can be blown away by the wind turbulence. All qualitative analysis of these synthetic data is only illustrative, because the simulation is too complex and too nonlinear to be represented by a simple qualitative/statistical relation. Changing the point of view is necessary here. We have a fast train of wind shock-waves which in a confined environment produces turbulence that blows away all small objects. The proposed wind turbulence model shows a new capability, the effective softattrition weapon against the smart adversary team of UAVs, which expresses fuzzy entanglement behavior.

\section{ACKNOWLEDGMent}

The authors are grateful to Dr. Tim Pattison, JOAD, for reviewing this manuscript and suggesting a number of clarifications that have significantly improved the overall quality of the paper.

\section{REFERENCES}

Chapra, S. and Canale, R. (2009). Numerical Methods for Engineers (6th Ed.), McGraw-Hill, New York.

Custers, B. (2016). The Future of Drone Use, Springer, New York.

Ivancevic, V., Reid, D. and Pilling, M. (in press). Mathematics of Autonomy: Mathematical Methods for Cyber-Physical-Cognitive Systems, World Scientific, Singapore (a 400-page monograph).

Ivancevic, V. (2010). Adaptive-Wave Alternative for the Black-Scholes Option Pricing Model, Cogn. Comput., 2, 17-30.

Ivancevic, V. (2011). Adaptive Wave Models for Sophisticated Option Pricing, J. Math. Finance, 1, 41-49.

Ivancevic, V. and Reid (2012). Turbulence and Shock-Waves in Crowd Dynamics, Nonlin. Dynamics, 68, 285-304.

Ivancevic, V. and Yue, Y. (2016). Hamiltonian Dynamics and Control of a Joint Autonomous Land-Air Opera-tion, Nonlin. Dynamics, 84, 1853-1865.

Schoellig, A.P., Mueller, F.L. and D’Andrea, R. (2012). Optimization based iterative learning for precise quadrocopter trajectory tracking. Auton. Robots, 33(1-2), 103-127. 